\title{
Total laparoscopic limited anatomical resection for centrally located hepatocellular carcinoma in cirrhotic liver
}

\author{
Cheng-Maw Ho $\cdot$ Go Wakabayashi $\cdot$ \\ Hiroyuki Nitta • Masahiro Takahashi • \\ Takeshi Takahara • Naoko Ito • Yasushi Hasegawa
}

Received: 15 March 2012/ Accepted: 14 September 2012/Published online: 12 December 2012

(C) The Author(s) 2012. This article is published with open access at Springerlink.com

\begin{abstract}
Background Limited anatomical liver resection for hepatocellular carcinoma (HCC) is complicated in cirrhotic patients with centrally located HCC and limited liver reserve. We present a case of total laparoscopic left medial and right ventroanterior sectionectomy performed using the intrahepatic Glissonian approach in a cirrhotic liver for curative resection of HCC.

Methods The patient was a 69-year-old man with a 6.5$\mathrm{cm}$-diameter HCC located at segments 4, 5, and 8 and which was compressing the middle hepatic vein (MHV). Child-Pugh class A liver cirrhosis was noted, and the 15-min retention rate for indocyanine green was $14 \%$. Preoperative surgical planning suggested the feasibility of limited anatomical subsegmental resection. The patient was placed in the supine position and 5 trocars were used for the procedure. The operation began with cholecystectomy, division of liver ligaments, and exposure of the right hepatic vein root and the umbilical Glissonian pedicles to the left medial segment. Parenchymal transection was performed using a laparoscopic harmonic scalpel and Cavitron Ultrasonic Surgical Aspirator until the MHV was reached. After exposing the ventral branches of the right
\end{abstract}

Electronic supplementary material The online version of this article (doi:10.1007/s00464-012-2624-6) contains supplementary material, which is available to authorized users.

C.-M. Ho · G. Wakabayashi $(\bowtie) \cdot$ H. Nitta $\cdot$ M. Takahashi ·

T. Takahara $\cdot$ N. Ito $\cdot$ Y. Hasegawa

Department of Surgery, Iwate Medical University School of

Medicine, 19-1 Uchimaru, Morioka, Iwate 020-8505, Japan

e-mail: gowaka@iwate-med.ac.jp

C.-M. Ho

Department of Surgery, National Taiwan University Hospital,

Taipei, Taiwan anterior Glissonian pedicle and dividing them, resection was continued along the demarcation line. Fissure veins draining to the MHV root were identified and divided. The MHV root was closed using an automatic stapler.

Results The operation time was $565 \mathrm{~min}$ and estimated blood loss was $665 \mathrm{ml}$; blood transfusion was not required. Pathological examination confirmed a moderately differentiated HCC with all resected margins free of malignancy. Postoperative recovery was uneventful and the patient was discharged on the postoperative day 7 . There was no tumor recurrence 18 months after the operation.

Conclusions Total laparoscopic left medial and right ventroanterior sectionectomy via the intrahepatic Glissonian approach is feasible for HCC in a cirrhotic liver with limited liver reserve. Preoperative planning is essential in order to compute successful hepatic function. Standardization of surgical techniques may aid in safely performing this procedure.

Keywords Limited anatomical resection - Laparoscopy · Hepatocellular carcinoma $\cdot$ Cirrhosis

Laparoscopic liver resection, which was first reported in 1992 [1], is gaining increasing acceptance and is often applied as the surgical treatment of hepatocellular carcinoma (HCC). A previous study conducted on a selected group of patients showed that the 5-year survival rate of patients who underwent laparoscopic HCC resection was comparable to that of patients who underwent open hepatic resection [2]. Compared to nonanatomic resection, anatomic resection for a single $\mathrm{HCC}$ is known to yield a more favorable outcome [3], which holds true also for anatomic subsegmentectomy [4]. However, cirrhosis is present in over $80 \%$ of patients with HCC [5]. Maintaining adequate 

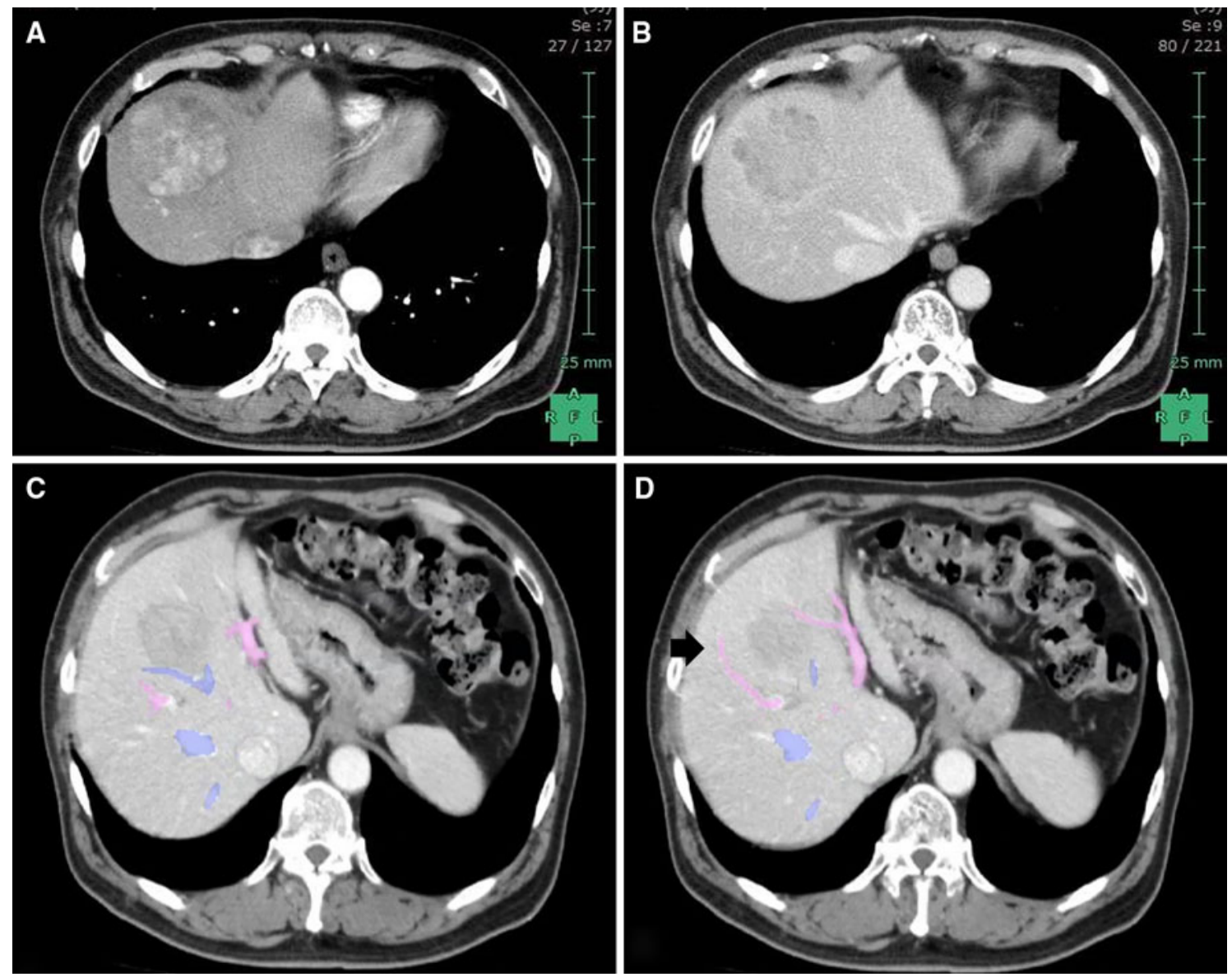

Fig. 1 Enhanced abdominal computed tomographic image showing A early arterial phase of HCC centrally located in the liver and $\mathbf{B}$, C compression of the MHV. D Ventral branch of the right anterior

portal trunk is pointed to by the arrow. Pink portal vein, blue hepatic vein (Color figure online)

liver function during resection is extremely important for patient survival [6]. In HCC patients, particularly those with a large HCC, it is difficult to safely perform laparoscopic anatomic resection with an adequate resection margin [7]. Therefore, laparoscopic liver resection for HCC is limited to highly selected cases [8].

Laparoscopic left medial and right anterior sectionectomy (central hepatectomy) has rarely been performed, with only nine cases reported to date [2, 9, 10]. Among these cases, two involved purely laparoscopic resection: in one case, an otherwise healthy 43-year-old man underwent liver resection for colorectal metastasis [9], and in the other case, the patient underwent resection for a small HCC [10]. To our knowledge, there is no report on totally laparoscopic left medial and right ventroanterior sectionectomy (segment 4 , subsegments 5 and 8). Here, we present the case of a patient with a large HCC who was successfully treated using limited anatomic resection of the left medial and right ventroanterior liver segments via an intrahepatic Glissonian approach.

\section{Patient and methods}

A 68-year-old man with a history of diabetes mellitus and hypertension, but without hepatitis $\mathrm{B}$ or $\mathrm{C}$ virus infection, was sonographically confirmed to have a large hepatic tumor during follow-up. He had undergone exploratory laparotomy for colon diverticulitis 7 years prior. Abdominal computed tomography revealed a $6.5-\mathrm{cm}$ liver mass, which showed typical HCC features of early arterial enhancement and portal venous washout, located at hepatic segments 4,5 , and 8 and compressing the middle hepatic vein (MHV, Fig. 1A-C). The tumor was supplied by the ventral branches of the right anterior Glissonian pedicle (Fig. 1D). Preoperative evaluation of the liver reserve revealed Child-Pugh class A liver cirrhosis and the 15 -min retention rate of indocyanine green $\left(\mathrm{ICG}_{15}\right)$ was $14 \%$. The serum levels of tumor markers for alphafetoprotein and protein induced by vitamin $\mathrm{K}$ absence or antagonist-II were $2.6 \mathrm{ng} / \mathrm{ml}$ and $132 \mathrm{mAU} / \mathrm{ml}$, respectively. Preoperative surgical planning using the three- 

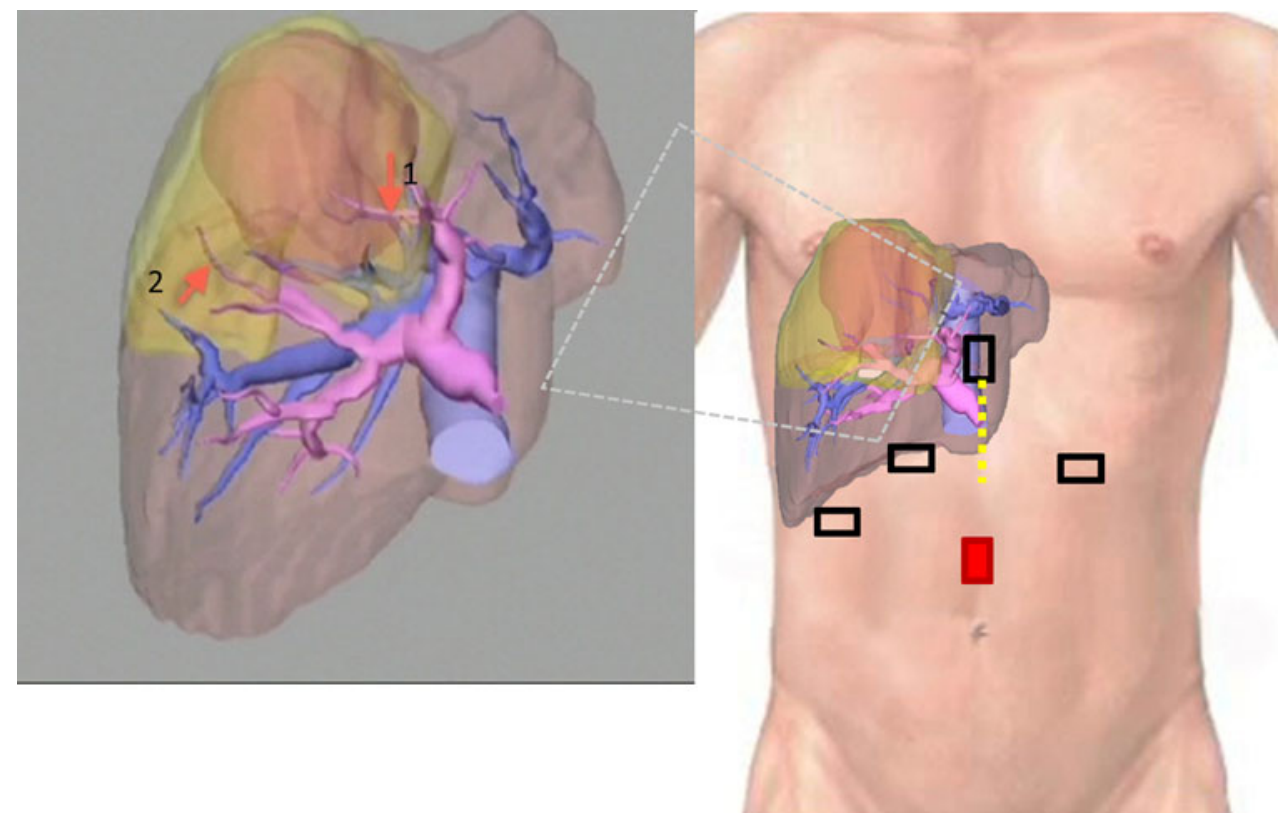

Fig. 2 Preoperative surgical planning using 3D liver volumetry and port setting. Calculated volumes of liver (coffee color) and tumor (orange) were 1,067 and $144 \mathrm{ml}$, respectively. The tumor compressed the MHV and occupied the parenchyma supplied by the S4 Glissonian (arrow 1) and the ventral branch (arrow 2) of the right anterior Glissonian pedicles. The proposed resection area, estimated at $260 \mathrm{ml}(24 \%$ total liver

dimensional volume analyzer Synapse Vincent ${ }^{\mathrm{TM}}$ (FUJIFILM Co., Japan) suggested the feasibility of limited anatomic subsegmental resection (Fig. 2) instead of central bisegmentectomy (which consisted of 493-ml volume, $46.2 \%$ total liver volume) according to Makuuchi's criteria [11].

We performed total laparoscopic resection of the left medial (segment 4) and right ventroanterior (subsegments 5 and 8) liver segments. The patient was placed in the supine position with the surgeon standing on the right side of the patient. Five trocars were used as shown in Fig. 2. The operation began with adhesiolysis and cholecystectomy. The right triangular and falciform ligaments were divided from the medial to the lateral side in order to avoid any damage to the inferior vena cava (IVC) and for safely locating the root of the right hepatic vein (RHV). Intraoperative sonographic examination was used to confirm the exact tumor location and its relationship to the major blood vessels. Then, parenchymal transection was performed along the medial side of the falciform ligament, thereby exposing the umbilical Glissonian pedicles, which included the arterial, portal, and bile duct branches of segment 4 and which were clipped and divided (Fig. 3A). The Cantlie line demarcation of the liver lobes was identified and the parenchymal resection was continued toward the right liver lobe using specialized surgical instruments (harmonic scalpel for superficial parenchyma and laparoscopic Cavitron Ultrasonic Surgical Aspirator [CUSA; Valleylab, volume), is shown in yellow, which covers the tumor completely. Five ports were required for totally laparoscopic left medial and right ventroanterior segmentectomy. The camera port is in red. All ports, except the left subcostal port, were $12 \mathrm{~mm}$ in diameter. The portal vein is shown in purple; the hepatic veins and IVC are in blue. Incision for minilaparotomy is shown as the yellow dotted line (Color figure online)

Boulder, $\mathrm{CO}$ ] for deep parenchymal tissue), until the level of the MHV (Fig. 3B). The ventral branches of the right anterior Glissonian pedicle were identified, clipped, and divided (Fig. 3C). Furthermore, the new demarcation line, which leaves segments 6 and 7 and dorsal subsegments 5 and 8 intact, appeared (Fig. 3D). Right ventroanterior sectionectomy was then performed. Meticulous transection was necessary to avoid massive bleeding from the transected surface. As the transection proceeded toward the root of the MHV, we encountered the drainage vein from segment 8 (fissure vein) and had to divide the vein. The root of the MHV was closed using an automatic stapler and intraoperative sonography was performed to confirm the integrity of the RHV root. The tumor specimen was removed via minilaparotomy (Fig. 4). Because slight bile spillage was observed from the exposed right anterior Glissonian pedicle, fibrin glue and sealant patch $\left(\mathrm{TachoSil}^{\circledR}\right.$, Nycomed, Linz, Austria) were applied topically to the surface of the bile duct for cover reinforcement after inserting a bile drainage tube (5-French ureteric catheter), which was placed into the remnant cystic duct by minilaparotomy as shown in Fig. 2.

\section{Results}

The operation time was 565 min, the estimated blood loss was $665 \mathrm{ml}$, and there was no need for blood transfusion. 

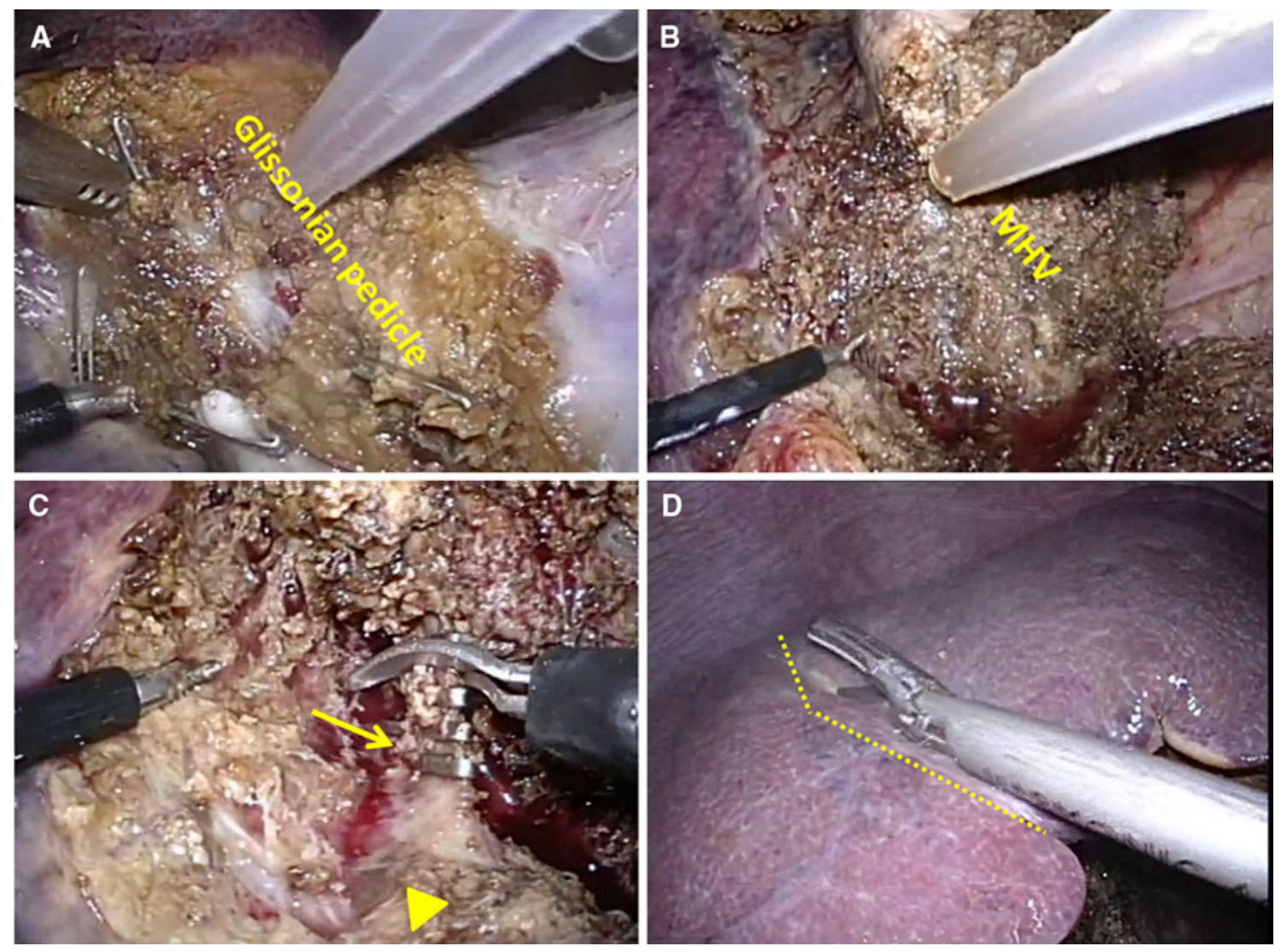

Fig. 3 The surgical strategy adopted during total laparoscopic medial and ventroanterior sectionectomy with intraoperative views of the major steps. A Left umbilical Glissonian pedicle, which was divided using a vascular endoscopic stapler. B Parenchymal transection of the left medial segment toward the right liver lobe. The MHV is shown. C The right ventral branch of the anterior Glissonian pedicle (arrow)

was divided using vascular endoscopic clips, leaving the dorsal branch intact (arrowhead). D The demarcation line appeared after dividing ventral branches of the right anterior Glissonian pedicle, which was shifted toward the right side from the Cantlie line (broken line)

Fig. 4 The resected specimen (weight $320 \mathrm{~g}$ ), showing a 6.5 cm-diameter HCC with negative resection margins

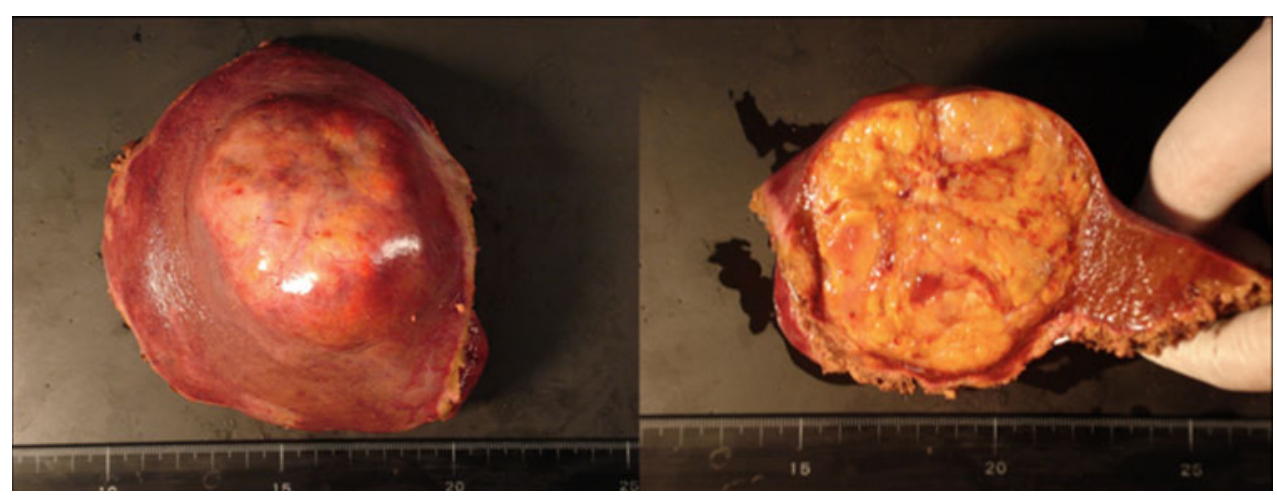

The bile drainage tube was removed on the postoperative day 4. Postoperative recovery was uneventful and the patient was discharged on the postoperative day 7. Pathological examination of the tumor specimen confirmed a stage II moderately differentiated HCC with negative resection margins. The patient showed no tumor recurrence during the follow-up 18 months after surgery.

\section{Discussion}

Laparoscopic surgery is popular because it offers benefits such as a small operative wound and a fast recovery. Anatomic liver resections such as right or left hepatectomy via laparoscopic procedures have been previously reported [2]. However, to our knowledge, only two case reports of 
pure laparoscopic central bisegmentectomy have been performed: one for a patient with liver metastasis [9] and the other for a patient with small HCC [10]. Here, we demonstrated the feasibility of performing total laparoscopic left medial and right ventroanterior sectionectomy for a $6.5-\mathrm{cm}$-diameter $\mathrm{HCC}$ in a Child-Pugh class A cirrhotic patient. The indications for performing laparoscopic liver resection could be extended to centrally located HCC in a mildly cirrhotic background, which is similar to that for open surgery, so that both anatomic liver resection and maintenance of liver reserve could be adequately achieved. Actuarial preoperative planning is essential to compute successful hepatic function. Further advancements in this technique may enable an increasing number of HCC patients to benefit from this procedure.

Left medial and right anterior sectionectomy is a safe and effective procedure for the treatment of centrally located HCC [12]. Although this procedure is technically demanding, a greater amount of nontumor liver parenchyma is preserved, which is important for the survival of patients with liver cirrhosis. While performing both open surgery and left medial and right anterior sectionectomy, the major difficulty is in safely dissecting the two parenchymal transection planes and in simultaneously preserving the vascular structures that drain or supply the remnant liver [10]. The same principles, though even much more challenging, apply for left medial and right ventroanterior sectionectomy. Detailed anatomical knowledge of the branches of the Glissonian pedicle to the right paramedian segments (segment 5 and 8) is key to performing limited anatomical subsegmental resection [13]. Parenchymal transection in open subsegmentectomy could be guided by ultrasonically guided portal vein puncture and injection of dye which marks the demarcation line [14]. In laparoscopic subsegmentectomy, which is difficult to do, the substitute maneuver is the identification and division of the intrahepatic Glissonian pedicles. Careful dissection and proper surgical control of all the vascular structures are critical for safe and successful resection, particularly in the hilum and the deep portion around the hepatic veins and IVC [10]. There were four critical steps for successful completion of this procedure: surgical control of the intrahepatic Glissonian pedicles (left medial and the ventral branch of the right anterior pedicle), identification of the MHV as parenchymal transection proceeded, division of fissure veins draining to the MHV, and division of the MHV root. Standardization of the surgical techniques is important for facilitating this procedure. Laparoscopic CUSA is very helpful for the detection and isolation of deep-seated vascular structures. Experience in performing a one-plane laparoscopic transection may help surgeons safely perform this two-plane laparoscopic transection. We eagerly anticipate further development of this procedure for increased chances of treatment for HCC patients.

\section{Conclusions}

Total laparoscopic left medial and right anterior sectionectomy is feasible for cirrhotic patients with $\mathrm{HCC}$ when residual liver volume is limited. Preoperative planning is essential in order to compute successful hepatic function. The intrahepatic Glissonian approach is useful for this complex procedure. Standardization of surgical techniques may help laparoscopic surgeons safely perform this challenging procedure.

Disclosures Drs. Cheng-Maw Ho, Go Wakabayashi, Hiroyuki Nitta, Masahiro Takahashi, Takeshi Takahara, Naoko Ito, and Yasushi Hasegawa have no conflicts of interest or financial ties to disclose.

Open Access This article is distributed under the terms of the Creative Commons Attribution License which permits any use, distribution, and reproduction in any medium, provided the original author(s) and the source are credited.

\section{References}

1. Gagner M, Rheault M, Dubuc J (1992) Laparoscopic partial hepatectomy for liver tumor (abstract). Surg Endosc 6:99

2. Nguyen KT, Gamblin TC, Geller DA (2009) World review of laparoscopic liver resection-2,804 patients. Ann Surg 250: $831-841$

3. Hasegawa K, Kokudo N, Imamura H, Matsuyama Y, Aoki T, Minagawa M, Sano K, Sugawara Y, Takayama T, Makuuchi M (2005) Prognostic impact of anatomic resection for hepatocellular carcinoma. Ann Surg 242:252-259

4. Eguchi S, Kanematsu T, Arii S, Okazaki M, Okita K, Omata M, Ikai I, Kudo M, Kojiro M, Makuuchi M, Monden M, Matsuyama Y, Nakanuma Y, Takayasu K, Liver Cancer Study Group of Japan (2008) Comparison of the outcomes between an anatomical subsegmentectomy and a non-anatomical minor hepatectomy for single hepatocellular carcinomas based on a Japanese nationwide survey. Surgery 143:469-475

5. El-Serag HB (2011) Hepatocellular carcinoma. N Engl J Med $365: 1118-1127$

6. Tanaka K, Shimada H, Matsumoto C, Matsuo K, Nagano Y, Endo I, Togo S (2008) Anatomic versus limited nonanatomic resection for solitary hepatocellular carcinoma. Surgery 143:607-615

7. Lee KF, Wong J, Cheung YS, Ip P, Wong J, Lai PB (2010) Resection margin in laparoscopic hepatectomy: a comparative study between wedge resection and anatomic left lateral sectionectomy. HPB (Oxford) 12:649-653

8. Santambrogio R, Aldrighetti L, Barabino M, Pulitanò C, Costa M, Montorsi M, Ferla G, Opocher E (2009) Laparoscopic liver resections for hepatocellular carcinoma. Is it a feasible option for patients with liver cirrhosis? Langenbecks Arch Surg 394: 255-264

9. Gumbs AA, Gayet B (2008) Totally laparoscopic central hepatectomy. J Gastrointest Surg 12:1153

10. Yoon YS, Han HS, Cho JY, Ahn KS (2009) Totally laparoscopic central bisectionectomy for hepatocellular carcinoma. J Laparoendosc Adv Surg Tech A 19:653-656

11. Makuuchi M, Kosuge T, Takayama T, Yamazaki S, Kakazu T, Miyagawa S, Kawasaki S (1993) Surgery for small liver cancers. Semin Surg Oncol 9:298-304 
12. Hu RH, Lee PH, Chang YC, Ho MC, Yu SC (2003) Treatment of centrally located hepatocellular carcinoma with central hepatectomy. Surgery 133:251-256

13. Cho A, Okazumi S, Makino H, Miura F, Shuto K, Mochiduki R, Tohma T, Kudo H, Matsubara K, Gunji H, Yamamoto H, Ryu M,
Ochiai T (2004) Anterior fissure of the right liver-the third door of the liver. J Hepatobiliary Pancreat Surg 11:390-396

14. Makuuchi M, Hasegawa H, Yamazaki S (1985) Ultrasonically guided subsegmentectomy. Surg Gynecol Obstet 161:346-350 